\title{
Comparative profiling between primary colorectal carcinomas and metastases identifies heterogeneity on drug resistance
}

\author{
Feng Luo ${ }^{1, *}$, Jinbang $\mathrm{Li}^{1,}{ }^{1}$, Shigang $\mathrm{Wu}^{1}{ }^{1}$, Xuefang $\mathrm{Wu}^{1}$, Meixiang Chen ${ }^{1}$, Xueyun \\ Zhong ${ }^{2}$, Kunping Liu ${ }^{1}$ \\ ${ }^{1}$ Department of Pathology, Qingyuan People's Hospital, Jinan University, Qingyuan 511518, China \\ ${ }^{2}$ Department of Pathology, Medical College, Jinan University, Guangzhou 510632, China \\ *These authors have contributed equally to this work \\ Correspondence to: Kunping Liu, email: gdaylkp@163.com \\ Xueyun Zhong, email: tzxy@jnu.edu.cn
}

Keywords: colorectal cancer, drug resistance, WNT signaling pathway, EMT, cancer stem cells

Received: March 14, 2016

Accepted: August 11, 2016

Published: August 24, 2016

\section{ABSTRACT}

\begin{abstract}
Metastases cause recurrence and mortality for patients with colorectal carcinomas (CRC). In present study, we evaluated heterogeneity on drug resistance and its underlying mechanism between metastatic and primary CRC.Immunohistochemical results from clinical tissue microarray (TMA) suggested that the expression concordance rates of cancer stem cells (CSCs) and drug resistance relative proteins between lymph-node metastatic and primary CRC foci were low. The apoptotic and proliferation indexes in metastasis CRC specimens were decreased compared with primary. In vitro experimental results indicated that the migration and invasion abilities were upregulated in metastatic cells SW620 compared with primary cells SW480, the cellular efflux ability and WNT/ $\beta$-catenin activity were also upregulated in SW620 cells. After 5-fluorouracil (5-Fu) treatment, the reduction in the proportion of cell apoptosis, CD133 and TERT expression levels in SW620 were lower than that in SW480 cells. Bioinformatics analysis in whole-genome transcriptional profiling results between metastatic and primary CRC cells suggested that differentially expressed genes were mainly centered on well-characterized signaling pathways including WNT/ $\beta$-catenin, cell cycle and cell junction. Collectively, heterogeneity of drug resistant was present between metastatic and primary CRC specimens and cell lines, the abnormal activation of WNT/ $\beta$-catenin signaling pathway could be a potential molecular leading to drug resistant ability enhancing in metastatic CRC cells.
\end{abstract}

\section{INTRODUCTION}

Colorectal cancer (CRC) is one of the most common malignancies in the world. Approximately $25 \%$ to $50 \%$ of colorectal cancer patients develop metastatic disease [1]. Once metastasis has occurred in CRC, a complete cure of the disease is unlikely. Colorectal liver metastasis (CLM), occurring in about $20 \%$ of CRC patients during the course of their treatment, is the most common distant metastasis from CRC $[2,3]$.

The phenotype of human CRC cells mainly depends on the interaction of genetic and environmental factors. Numerous researches have pointed out that the heterogeneity was formed during and after the process of epithelial-mesenchymal transition (EMT) in CRC cells
[3-5]. Thiruvengadam and colleagues reported that Zeb-1 and other regulators of EMT maintain drug resistance in human pancreatic cancer cells [6]. Moreover, the process of EMT leads to great increases in the number of self-renewing cells that can initiate the seeding of mammospheres, which raises the possibility for achieving EMT process, at the same time, may also impart selfrenewal and multidrug resistance capabilities to metastatic cancer cells [7].

Chemotherapy has synergistic effects with radiotherapy and surgery and play vital roles in combined therapy for CRC $[8,9]$. Chemotherapy insensitivity and poor prognosis are biological characteristics of metastases CRC [10], but little is known about the biological behaviors differences between primary and metastatic 
CRC, especially at difference of drug resistance. Therefore, there is a need for better understanding of the molecular mechanisms underlying the metastatic phenotype that may provide information leading to the development of drugs to control or prevent metastatic disease.

In present study, to a much better understanding of biological differences between primary and metastatic CRC, we used high-throughput DNA microarrays to compare differentially expressed genes of primary and metastatic CRC cells. The migration and invasion abilities difference between primary CRC cells SW480 and metastatic cells SW620 were determined through transwell and boyden chamber assays. We found that the ability of drug resistant in metastatic cells SW620 was greater than primary colorectal cancer cells SW480 owing to cancer stem cells and drug resistance relative proteins activation, which partially reconfirmed by the clinical TMA IHC assay.

\section{RESULTS}

\section{Comparison of the CSCs and drug resistance relative proteins between primary $\mathrm{CRC}$ and colorectal lymphatic metastasis specimens}

To gain insight into the expression level of CSCs and drug resistance relative proteins in $\mathrm{CRC}$, we used the clinical tissue microarray to assess these protein expression levels between primary CRC and colorectal lymphatic metastasis specimens. The expression level of EpCAM (Membrane), E-cadherin, MRP, CD133 and Cyclin D1 were significantly upregulated in primary $\mathrm{CRC}$ foci compared with the corresponding lymph-node metastatic foci, whereas CD44v6 was downregulated in primary CRC foci (Figure $1 \mathrm{~A}$ and $1 \mathrm{~B}, p<0.05$ ). In addition, although there was no significant difference in the expression level of $\beta$-catenin between primary $\mathrm{CRC}$ and colorectal lymphatic metastasis specimens overall, the ratio of high expression $(+++)$ of $\beta$-catenin was signifcantly higher in lymph-node metastatic foci $(22.03 \%)$ compared with the corresponding primary CRC foci (10.16\%, Figure $1 \mathrm{~A}$ and $1 \mathrm{~B})$.

The concordance rates of the CSCs and drug resistance relative proteins between the primary $\mathrm{CRC}$ foci and corresponding lymph-node metastatic foci were showed in Figure 1C. The concordance rates were low, and there was only one protein, CD44v6, with over $50 \%$ concordance rate. To a better understanding the relationships of these CSCs and drug resistance relative proteins between the primary CRC foci and corresponding lymph-node metastatic foci, hierarchical clustering was generated by $\mathrm{R}$ programming language based on IHC results. Statistical analysis revealed that the expression level of E-cadherin was positively correlated with MRP and EpCAM expression in both primary CRC foci and corresponding lymphnode metastatic foci (Figure 1D and 1E, Table 1 and 2).
Meanwhile, the expression level of EpCAM (Membrane), E-cadherin and MRP were significantly higher in primary CRC foci compared with the corresponding lymph-node metastatic foci (Table 1 and 2, $p<0.001$ ).

\section{Comparison of the proliferation and apoptotic index between primary $\mathrm{CRC}$ and colorectal lymphatic metastasis specimens}

The apoptosis of CRC specimens was examined using TUNEL staining assay in CRC specimens, the apoptotic index of colorectal lymphatic metastasis specimens was significantly decreased compared to primary CRC specimens (Figure 2A, $p<0.001$ ). The proliferation index of CRC specimens was also examined using Cyclin D1 staining assay in CRC specimens (Figure 2B), we found that the proliferation index of colorectal lymphatic metastasis specimens was significantly decreased compared to primary CRC specimens $(p<0.05)$.

\section{The adhesive activity was downregulated, the efflux rate of $\mathrm{Rh} 123$ and WNT/B-catenin activity were upregulated in metastatic cells SW620}

The migration and invasion abilities difference between SW480 and SW620 cells were determined through transwell assays and boyden chamber assays. As shown in Figure 3A, the migration and invasion abilities of SW620 cells were significantly greater than SW480 cells $(p<0.05)$. Detection of the key components of cell migration and invasion pathways by western blotting demonstrated that compared to SW480 cells, the expression levels of CD44v6 and MMP-9 were elevated, while the level of E-cadherin was decreased in SW620 cells (Figure 3B, $p<0.05$ ). These results demonstrated that metastatic cells SW620 have greater migration and invasion abilities than primary colorectal cancer cells SW480.

To examine the P-gp function between SW480 and SW620 cells, the fluorescence intensity of Rh123 was measured by flow cytometry and the efflux rate of Rh123 was calculated. As shown in Figure 3C, the efflux rate of Rh123 was higher in SW620 cells than that in SW480 cells $(p<0.05)$. More interestingly, western blot analysis of $\beta$-catenin proteins in cytoplasmic and nuclear displayed that $\beta$-catenin was upregulated in the nuclei of SW620 compared to SW480 cells (Figure 3D, $p<0.05$ ). Immunofluorescence analysis verified that $\beta$-catenin was more favored distributed in the nuclei of SW620 cells compared with SW480 cells (Figure 3E).

\section{Cancer stem cells and drug resistance relative proteins activation lead to metastatic cells SW620 drug resistant ability enhancing}

After incubated with 5-Fu, the apoptosis induced by $5-\mathrm{Fu}$ of SW480 and SW620 cells was examined 
using flow cytometry after Annexin V-FITC/PI staining (Figure 4A). We found that $5-\mathrm{Fu}$ could significantly increase the apoptosis in SW480 compared to SW620 cells (Figure 4A, $p<0.05$ ). The apoptosis induced by $5-\mathrm{Fu}$ of CRC cells was also examined using TUNEL staining assay. Similarly, we found that 5-Fu could significantly increase the apoptosis in SW480 compared to SW620 cells (Figure 4B, $p<0.05$ ). These results suggested that

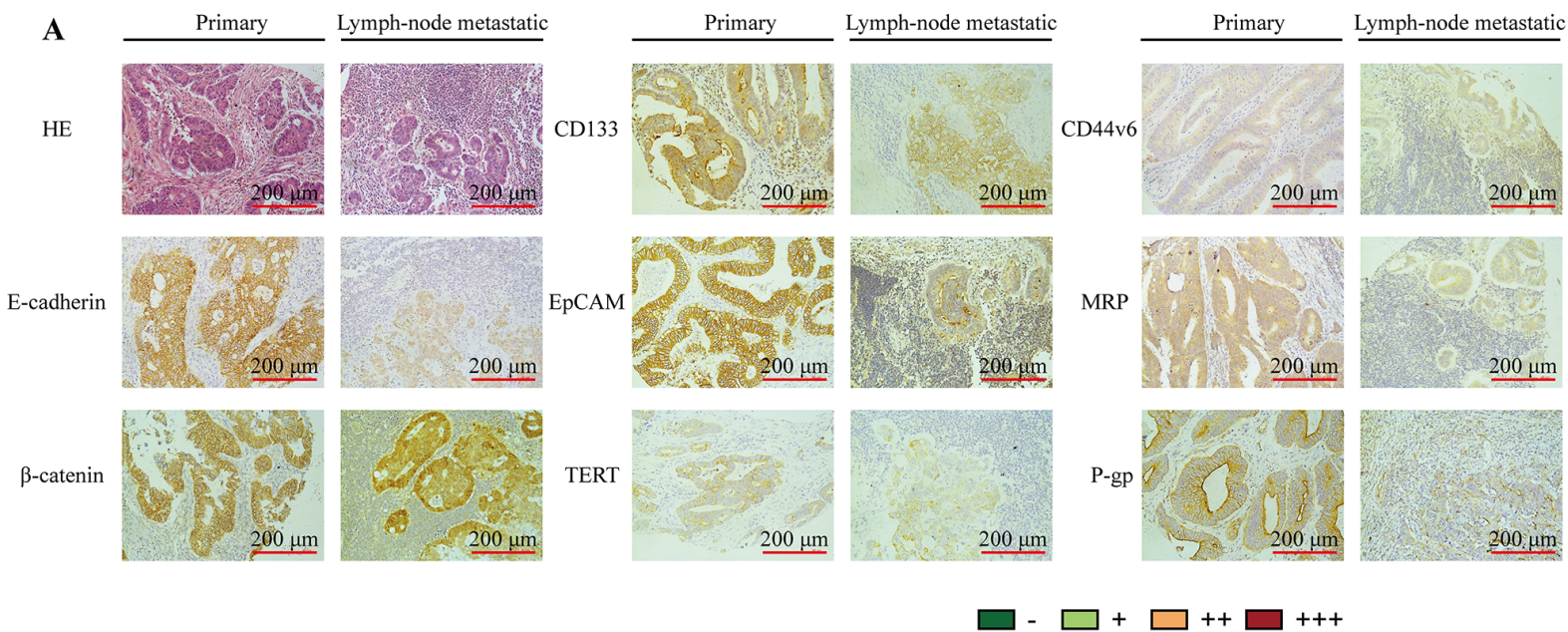

B Related-Samples Wilcoxon Signed Rank Test

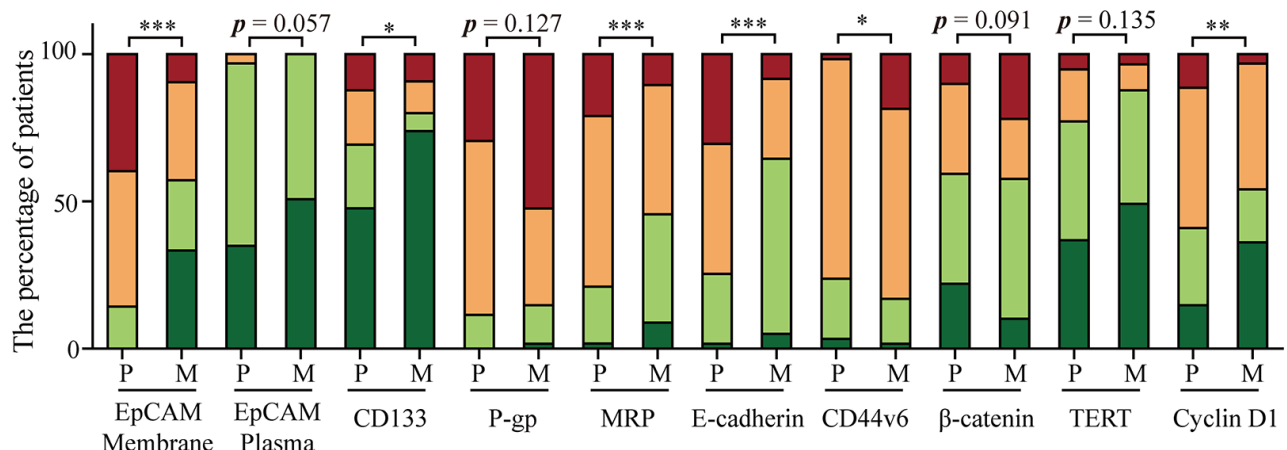

C

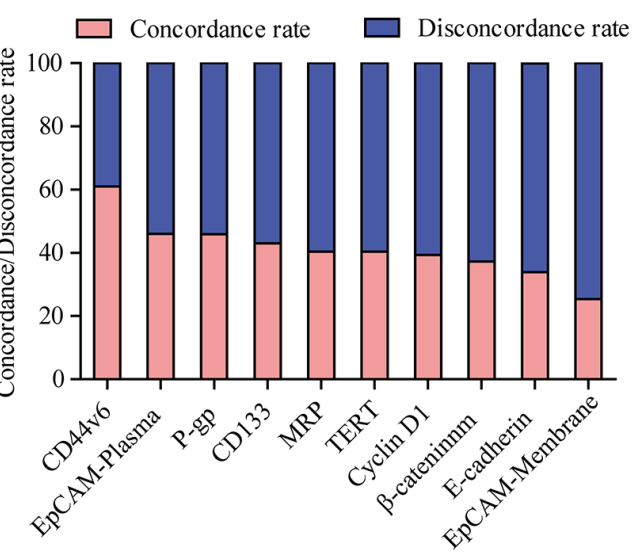

D

E-cadherin MRP MRP E-cadherin
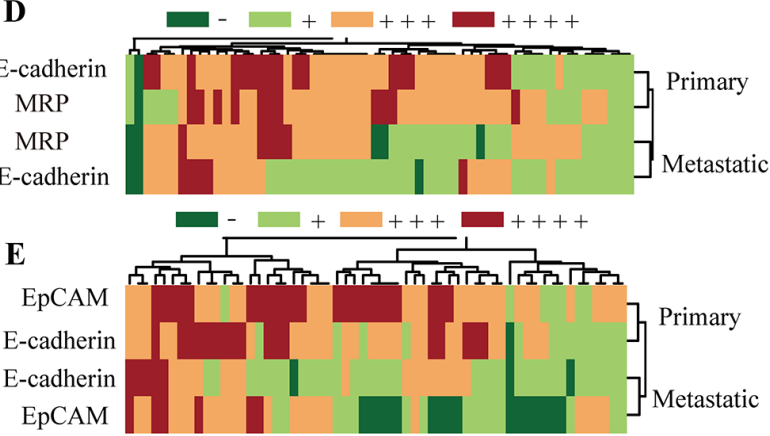

$\mathbf{E}$

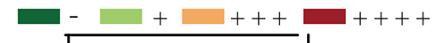

Figure 1: IHC staining assay on primary CRC and colorectal lymphatic metastasis specimens. A. Representative IHC of CRC samples, showing the expression level of EpCAM, CD133, P-gp, MRP, E-cadherin, CD44v6, $\beta$-catenin and TERT between primary CRC and colorectal lymphatic metastasis groups. B. The percentage of patients with the negative $(-)$, weak $(+)$, moderate $(++)$ and high $(+++)$ expression of CSCs and drug resistance relative proteins in primary CRC and colorectal lymphatic metastasis groups. P, primary. M, lymphatic metastasis. C. The concordance/disconcordance rate of CSCs and drug resistance relative proteins between primary CRC and colorectal lymphatic metastasis groups. D. Hierarchical clustering of the E-cadherin and MRP protein expression levels was generated by R programming language based on IHC results in primary CRC and colorectal lymphatic metastasis groups. E. Hierarchical clustering of the E-cadherin and EpCAM protein expression levels was generated by R programming language based on IHC results in primary CRC and colorectal lymphatic metastasis groups. High and low expressed proteins are shown by red and green, respectively. Each bar represents the means \pm SD. ${ }^{*} p<0.05, * * p<0.01, * * * p<0.001$. 
Table 1: Immunohistochemical analysis of E-cadherin and MRP in CRC in vivo

\begin{tabular}{lcc}
\hline & Primary-E-cadherin & Metastatic-MRP \\
\hline Primary-MRP & $p<0.01, r=0.353^{\mathrm{a}}$ & $p<0.001^{\mathrm{b}}$ \\
Metastatic-E-cadherin & $p<0.001^{\mathrm{b}}$ & $p<0.05, r=0.278^{\mathrm{a}}$ \\
\hline
\end{tabular}

a'Spearman's correlation Test, ${ }^{b}$ Related-Samples Wilcoxon Signed Rank Test

Table 2: Immunohistochemical analysis of E-cadherin and EpCAM in CRC in vivo

\begin{tabular}{lcc}
\hline & Primary-EpCAM & Metastatic-E-cadherin \\
\hline Primary-E-cadherin & $p<0.05, r=0.269^{\mathrm{a}}$ & $p<0.001^{\mathrm{b}}$ \\
Metastatic-EpCAM & $p<0.001^{\mathrm{b}}$ & $p<0.05, r=0.300^{\mathrm{a}}$ \\
\hline
\end{tabular}

aSpearman's correlation Test, ${ }^{b}$ Related-Samples Wilcoxon Signed Rank Test

metastatic cells SW620 have greater drug resistant ability than primary colorectal cancer cells SW480.

To investigate the underlying mechanisms leading to SW620 drug resistant ability enhancing, we assessed whether these effects of SW620 cells was due to cancer stem cells and drug resistance relative proteins activation. After either treated with 5-Fu or control for $24 \mathrm{~h}$, the effect of 5-Fu on the proportion of CD133+ of SW480 and SW620 cells was examined using flow cytometry. As shown in Figure 4C, the proportions of CD133+ SW480 cells were significantly decreased compared to SW620 cells $(p<0.05)$. As shown in Figure 4D, following $24 \mathrm{~h}$ treatment of 5-Fu, the expression level of TERT was downregulated in SW480 cells, whereas these effects were slight in SW620 cells. In addition, the expression levels of EpCAM were downregulated following $24 \mathrm{~h}$ treatment of 5-Fu in both SW480 and SW620 cells (Figure 4D, $p<0.05)$.

\section{Screening differentially expressed genes based on microarray data between metastatic and primary CRC}

In order to survey the differentially expressed genes between metastatic and primary CRC, we download cDNA microarrays from GEO database (No. GSE22834) and performed supervised analysis using SAM and identified 1,067 unique genes with aberrant expression in liver metastatic CRC compared to primary CRC. Functional annotations of the differentially expressed genes were generated using the GenCLiP 2.0 online tool.

As shown in Figure 5A, heatmap showing the differentially expressed genes and literature profiles-based keywords based on GenCLiP 2.0 online tool analyzed between metastatic and primary $\mathrm{CRC}$. With the results of clustering, the 16 highest-ranking literature profilesbased keywords of primary CRC and liver metastases comparison were listed in Figure 5B. We screened the most relevant genes associated with these keywords. Gene-gene interaction network of these 34 genes were generated and showed in Figure 5C and Table 3 using the GenCLiP 2.0 online tool.

\section{Screening differentially expressed genes based on microarray data between SW480 and SW620 cells}

Next, we extracted total RNA and conducted cDNA microarrays analysis (Roche, NimbleGen) between CRC cells derived from human primary colorectal cancer cells SW480 and their metastatic cells SW620. We then performed supervised analysis using SAM and identified 1,362 unique genes with aberrant expression in SW620 when compared to SW480 cells. Functional annotations of the differentially expressed genes were generated using the GenCLiP 2.0 online tool.

As shown in Figure 6A, heatmap showing the differentially expressed genes and literature profiles-based keywords based on GenCLiP 2.0 online tool analyzed between SW480 and SW620 cells. With the results of clustering, the 16 highest-ranking literature profiles-based keywords of SW480 and SW620 cells comparison were listed in Figure 6B. We screened the most relevant genes associated with these keywords. Gene-gene interaction network of these 56 genes were generated and showed in Figure 6C using the GenCLiP 2.0 online tool.

\section{Comparison of the differentially expressed genes between CRC primary-metastasis tissues and SW480-SW620 cell lines}

As noted above, microarray screening analysis found that a total number of 1,067 genes were differentially expressed between the metastatic and primary CRC, while 1,362 genes were differentially expressed between SW480 and SW620 cells. As shown in Figure 7A, the overlapping 
differentially expressed genes between the CRC tissues and cell lines were 97 genes; however, the overlapping among the top 10 highest-ranking keywords between the CRC tissues and cell lines were 6 (Figure 7A).

The differentially signaling pathways were analyzed using the GenCLiP 2.0 online tool. As shown in Figure $7 \mathrm{C}$, the histogram showing the differentially signaling pathways between metastatic and primary CRC. The various aberrant signaling pathways between metastatic and primary $\mathrm{CRC}$ indicated that heterogeneity was present among different tumors as well as between primary and metastatic CRC foci. The differentially signaling pathways between SW480 and SW620 cells were also showed in Figure 7B.

\section{DISCUSSION}

Metastases cause significant recurrence and mortality for patients with $\mathrm{CRC}$, and the survival rates with these case remain unacceptably low $[1,2,10]$. The process of EMT often considered being the switch that allows tumor cells to acquire the capacity to invade and ultimately metastasize to distant sites $[11,12]$. Numerous researches have pointed out that the genetic heterogeneity was present among different tumors as well as between primary and metastatic cancer cells $[13,14]$. However, the biological differences between primary and metastatic $\mathrm{CRC}$, especially at difference of drug resistance is still largely unknown. Therefore, elucidation of molecular mechanism underlying the metastatic phenotype is critical for the development of potential therapeutic agents for CRC.
DNA microarrays is a recent development and application of human genome and high-throughput technology, allow us to simultaneously examine thousands of genes, provided a promising way to much better understanding of CRC carcinogenesis [15-17]. The overlapping differentially expressed genes between the CRC tissues and cell lines were rare, but the overlap ratio among these top 10 highest-ranking keywords was $60 \%$. These results suggested that although differentially expressed genes are dissimilarity, the functions of these genes are mainly similar between CRC tissues and cell lines. To compare the biological behavior difference between primary and metastatic CRC cells, the migration and invasion abilities difference between SW480 and SW620 cells were determined through transwell and boyden chamber assays. Our findings reveal that metastatic CRC SW620 cells have greater migration and invasion abilities than primary SW480 cells, the relative proteins CD44v6 and MMP-9 were upregulated, E-cadherin was downregulated in SW620 cells. These results consistent with Palmieri and Kubens' experimental results [18, 19].

In present study, the immunohistochemistry results showed that the expression level of drug resistance and stem cell related proteins in metastatic and primary CRC foci were different. The concordance rates of these proteins between the lymphatic metastatic CRC foci and primary foci were low, and there was only one protein, CD44v6, with over $50 \%$ concordance rate. In addition, the apoptotic and the proliferation index in lymphatic metastasis CRC foci were decreased compared with primary foci. These results showed that the heterogeneity at difference expression level of drug resistance and stem cell related

A

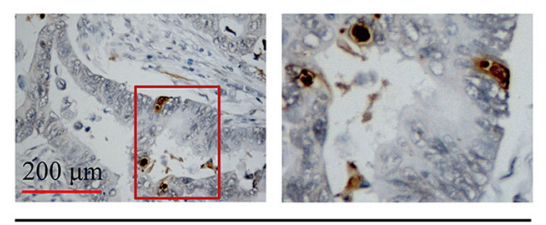

Primary

B

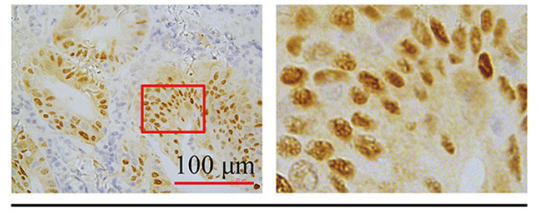

Primary

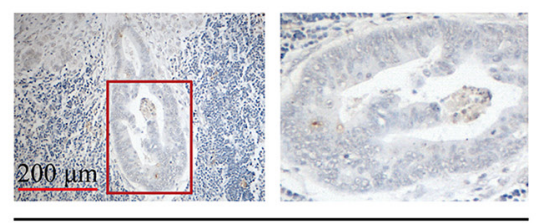

Lymph-node metastatic

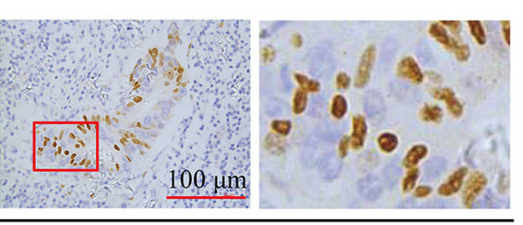

Lymph-node metastatic
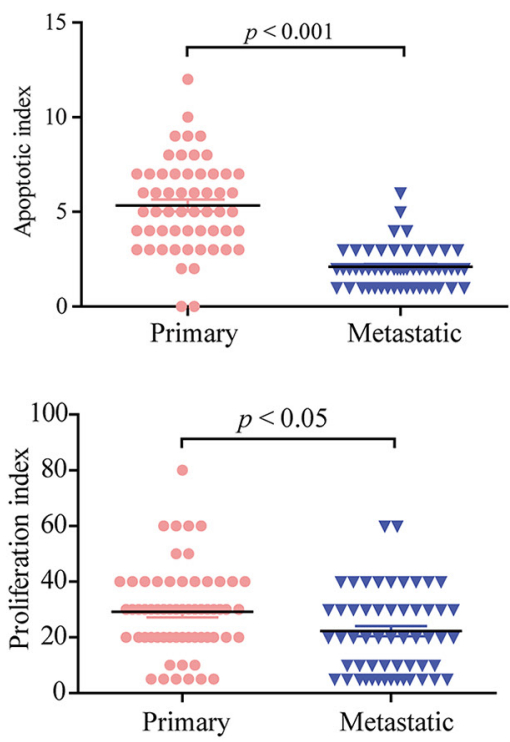

Figure 2: TUNEL and Cyclin D1staining assays on primary CRC and colorectal lymphatic metastasis specimens. A. Left, TUNEL staining assay on primary CRC and colorectal lymphatic metastasis specimens. Right, the apoptotic index in primary and metastatic groups. B. Left, Cyclin D1 staining assay on primary CRC and colorectal lymphatic metastasis specimens. Right, the proliferation index in primary and metastatic groups. Each bar represents the means \pm SD. ${ }^{*} p<0.05, * * p<0.01, * * * p<0.001$. 
proteins was present between the metastatic and primary CRC foci, suggested that biological behaviors between metastatic and primary cells may be different. In vitro, we found that the efflux rate of Rh123 was upregulated in metastasis CRC SW620 cells than primary SW480 cells. According to the flow cytometry and TUNEL staining experimental results, we found that reduction in the proportion of apoptosis in SW620 cells induced by 5-Fu treatment were lower than that in SW480 cells. After treated with $5-\mathrm{Fu}$, reduction in the proportion of CD133 and TERT protein expressions in SW620 cells were lower than SW480 cells. These results indicated that metastatic cells with such characteristics are partly responsible for its drug resistant ability enhancing. Perhaps this explains in part why the lower remission and higher recurrent were happened to patients with metastasis after chemotherapy treatment.
Analysis results from GenCLiP 2.0 suggested that WNT pathway was involved in aberrant signaling pathways between metastatic and primary $\mathrm{CRC}$, as well as between SW480 and SW620 cells. The main oncoprotein in colorectal cancer is the WNT pathway effector $\beta$-catenin, which transportation to the nucleus and overactivation due to genic mutations in the APC, Axin, CKI, and GSK-3 $\beta$ in most cases [20-25]. Aberrant WNT signaling pathway is associated with a wide array of tumor types and plays an important role in the maintenance of stemness of CSCs and drug resistant ability [26-31]. Our experimental results show that $\beta$-catenin was upregulated and accumulated in the nuclei, whereas E-cadherin was downregulated in SW620 compared with SW480 cells. Typically, E-cadherin and $\beta$-catenin located at the cell membranes and constituted intercellular junctions [32-36]. During the process of
A

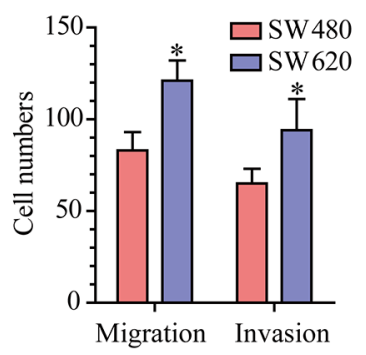

B
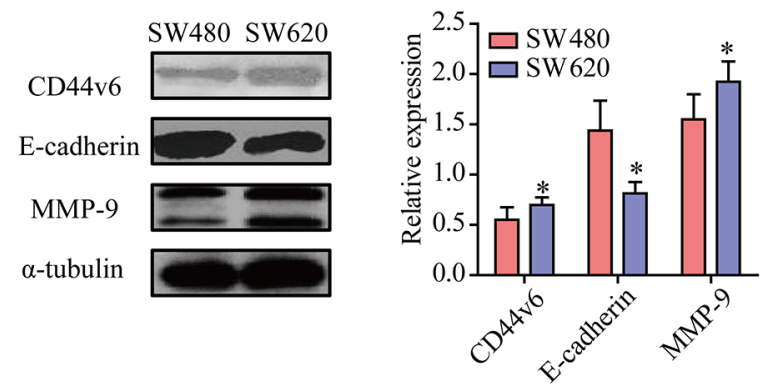

C
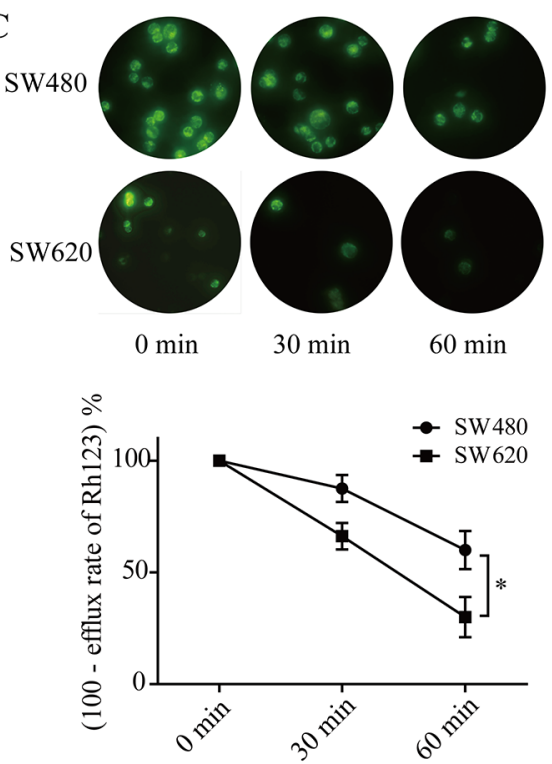

D

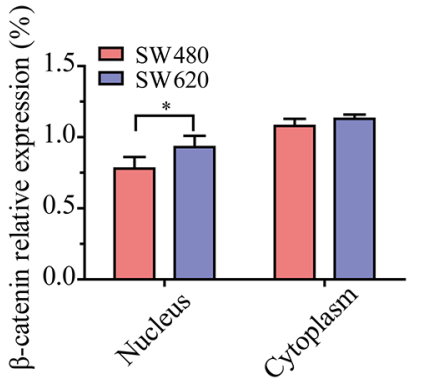

$\mathbf{E}$

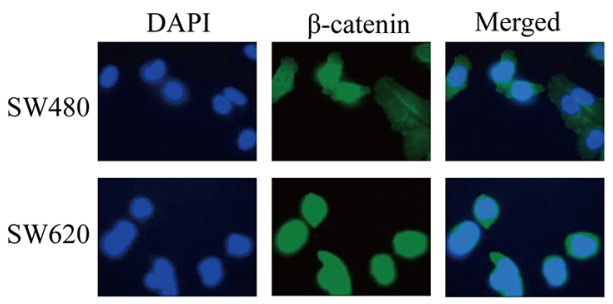

Figure 3: Comparison of adhesive activity, P-gp function and WNT/ $\beta$-catenin activity between SW480 and SW620 cells. A. Transwell assays and boyden chamber assays evaluated the migration and invasion abilities of SW480 and SW620 cells. B. Western blot analyzed of the expression levels of CD44v6, MMP-9 and E-cadherin in SW480 and SW620 cells. Each bar represents the means \pm SD. C. The fluorescent signals of Rh123 before and after 5-Fu treatment were observed by fluorescent microscope (200X) in SW480 and SW620 cells. D. Western blot analysis the expression level of $\beta$-catenin protein in cytoplasmic and nuclear of SW480 and SW620 cells. Values are expressed as protein/ $\alpha$-tubulin or protein/Histon. The density of the protein band was quantitated using Quantity One software. The data are expressed as mean $\pm \mathrm{SD}$ of three experiments. * $p<0.05$ compared with the SW480 cells. E. Immunofluorescence (400X) was done to visualize the expression and nuclear accumulation of $\beta$-catenin between SW480 and SW620 cells. 
EMT, the E-cadherin/ $\beta$-catenin complex breakdown, accompanying with $\beta$-catenin translocation into nuclei and WNT signaling pathway over activation in CRC [37-41]. In addition, our previous research shows that the WNT signaling pathway plays an important role in drug resistance in colon cancer cells by alternating the expression level of CSC markers and drug resistance relative proteins [42, 43]. Junlin and colleagues also reported that the activation of $\beta$-catenin and Akt pathways by Twist are critical for the maintenance of EMT associated cancer stem cell-like characters [44].

In conclusion, we show for the first time that the heterogeneity on drug resistance was present between metastatic CRC foci and primary foci and cell lines, suggested that the molecular pathological diagnosis and follow-up clinical therapies for primary CRC foci may not be justly suitable for the corresponding metastatic foci. $\mathrm{WNT} / \beta$-catenin signaling pathway could be a potential
A

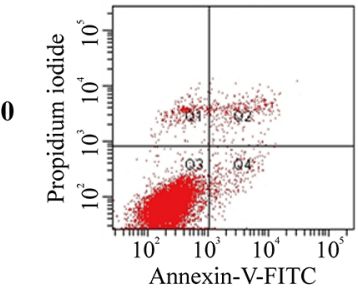

SW480
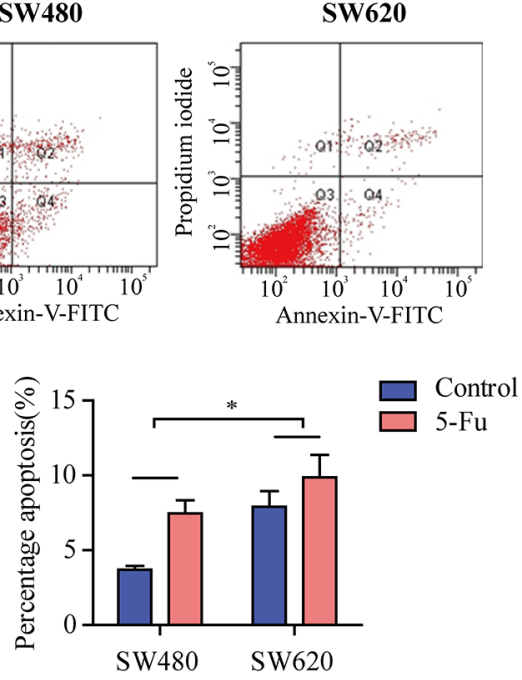

C

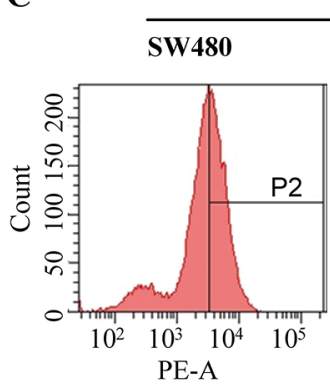

0

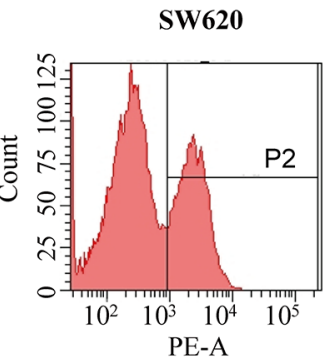

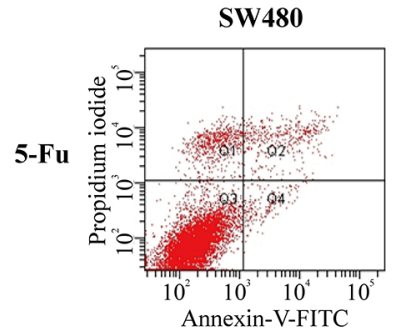

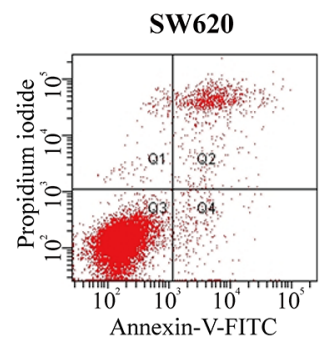

B

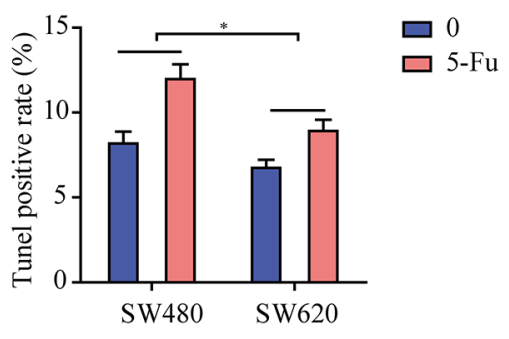

5-Fu

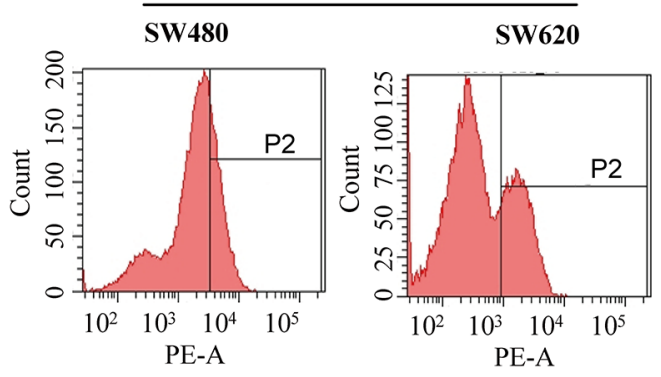

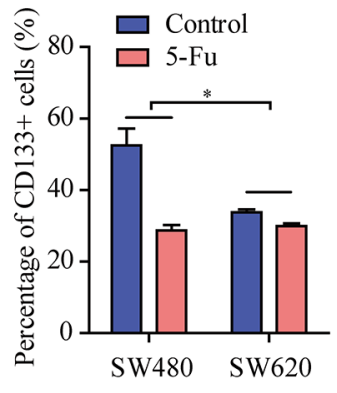
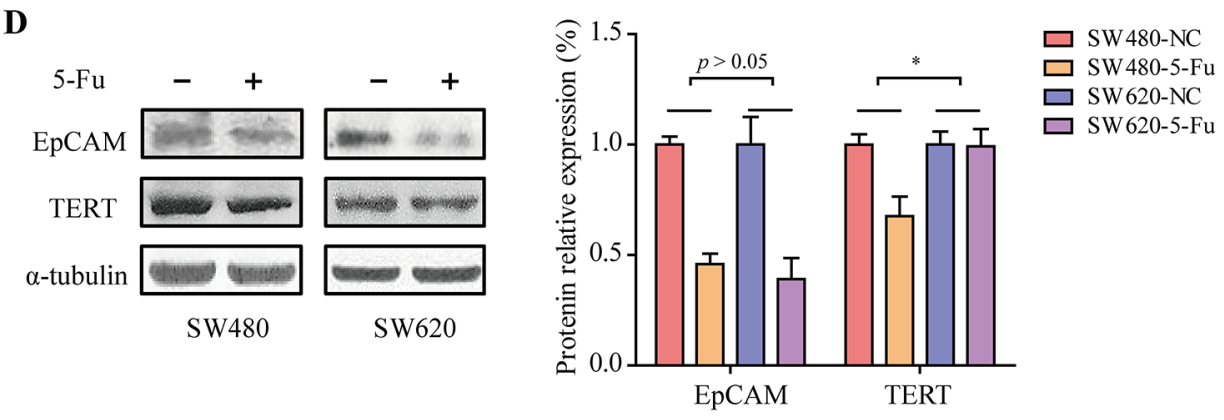

Figure 4: The effects of 5-Fu on cancer stem cells and drug resistance relative proteins of SW480 and SW620 cells. A. The rate of Annexin V positive cells before and after 5-Fu treatment in SW480 and SW620 cells. All values are presented as mean \pm SD of three experiments. B. CRC cells cultured in the presence of $10 \%$ serum on coverslips were kept in the absence and presence of $50 \mu \mathrm{mol} / \mathrm{L}$ 5-Fu for 3 days, at which point the cells were fixed and processed for TUNEL staining, to detect cells under-going programmed cells death. C. Flow cytometric analyzed the percentage of CD133+ cells when treated with 5-Fu or control in SW480 and SW620 cells. D. Expression levels of TERT and EpCAM in SW480 and SW620 cells before and after treatment with 5-Fu for 24h. * $p<0.05$. 


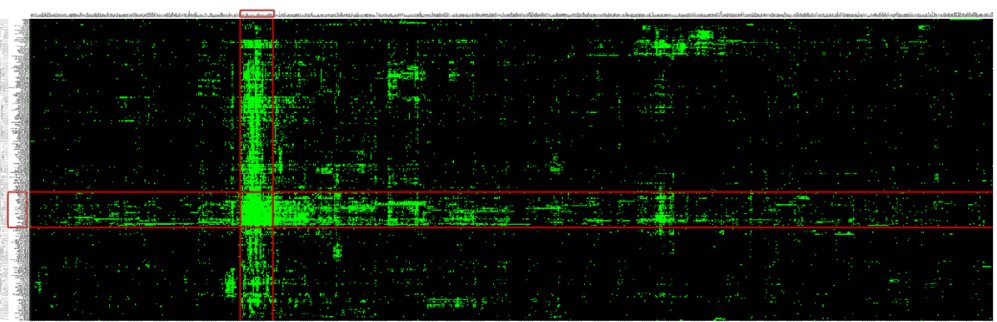

B

$\mathrm{C}$

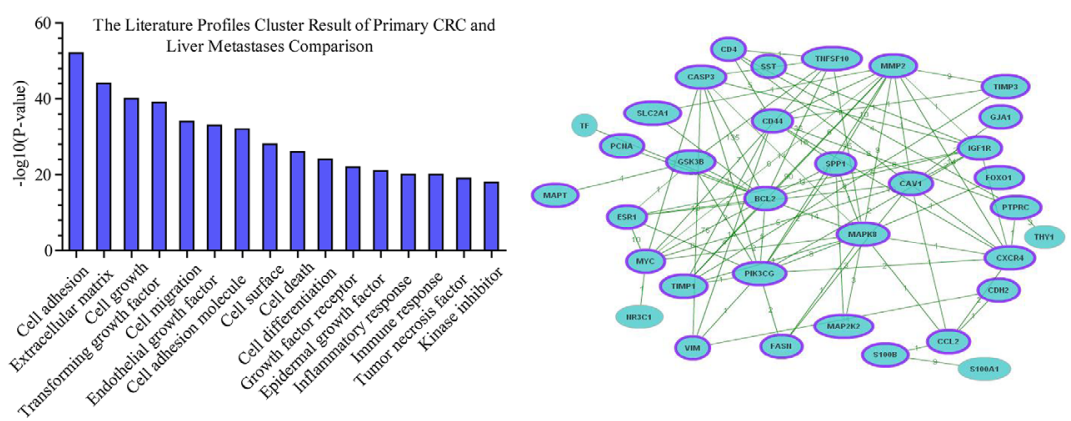

Figure 5: Functional annotations of the differentially expressed genes based on GenCLiP 2.0 online tool analyzed between CLM and primary CRC. A. Heatmap showed the clustering result generated from differentially expressed genes and literature profiles-based keywords based on GenCLiP 2.0 online tool analysis between CLM and primary CRC. Each row and column in the heat map representation is literature profiles-based keywords and differentially expressed genes, respectively. B. The literature profilesbased keywords cluster result of primary CRC and liver metastases comparison. C. Gene-gene interaction network of primary CRC and liver metastases comparison generated using the GenCLiP 2.0 online tool. The purple band mean genes in the network related with CRC according to the GenClip 2.0 literature data mining results. Related genes shown with purple band.

Table 3: The key differentially expressed genes between CLM and primary CRC

\begin{tabular}{lcc}
\hline Gene & Co-genes & Gene name \\
\hline BCL2 & 14 & B-cell CLL/lymphoma 2 \\
MAPK8 & 13 & Mitogen-activated protein kinase 8 \\
MMP2 & 12 & Matrix metallopeptidase 2 \\
PIK3CG & 12 & Phosphatidylinositol-4,5-bisphosphate 3-kinase catalytic subunit gamma \\
CD44 & 8 & CD44 molecule (Indian blood group) \\
CXCR4 & 8 & Chemokine (C-X-C motif) receptor 4 \\
IGF1R & 8 & Insulin like growth factor 1 receptor \\
MYC & 8 & Caspase 3 \\
CASP3 & 7 & Caveolin 1 \\
CAV1 & 7 & Estrogen receptor 1 \\
ESR1 & 6 & Secreted phosphoprotein 1 \\
SPP1 & 6 & TIMP metallopeptidase inhibitor 1 \\
TIMP1 & 6 & Tumor necrosis factor superfamily member 10 \\
TNFSF10 & 6 & Chemokine (C-C motif) ligand 2 \\
CCL2 & 5 & Glycogen synthase kinase 3 beta \\
GSK3B & 5 &
\end{tabular}

Gene names are according to the National Center for Biotechnology Information. Co-genes: Co-occurrence of gene pair and keyword(s) in Literature Mining Gene Networks. 


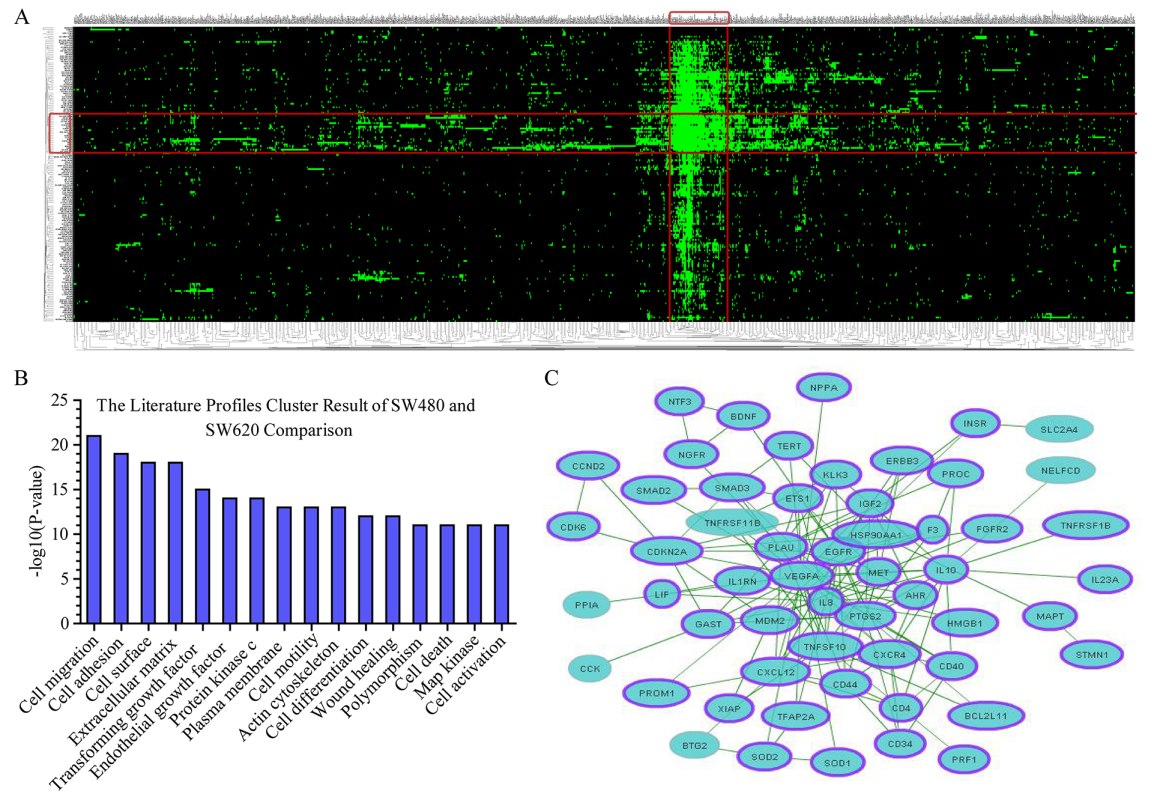

Figure 6: Functional annotations of the differentially expressed genes based on GenCLiP 2.0 online tool analyzed. A. Heatmap showed the clustering result generated from differentially expressed genes and literature profiles-based keywords based on GenCLiP 2.0 online tool analysis between SW480 and SW620 cells. Each row and column in the heat map representation is literature profiles-based keywords and differentially expressed genes, respectively. B. The literature profiles-based keywords cluster result of SW480 and SW620 comparison. C. Gene-gene interaction network of SW480 and SW620 comparison generated using the GenCLiP 2.0 online tool. The purple band mean genes in the network related with CRC according to the GenClip 2.0 literature data mining results. Related genes shown with purple band.

A
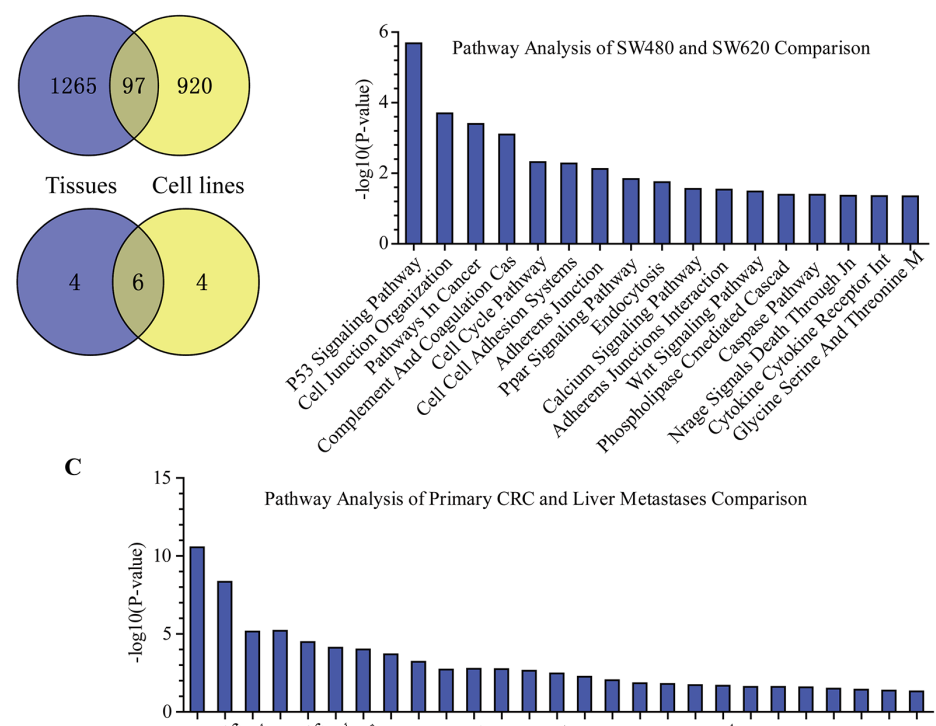

B

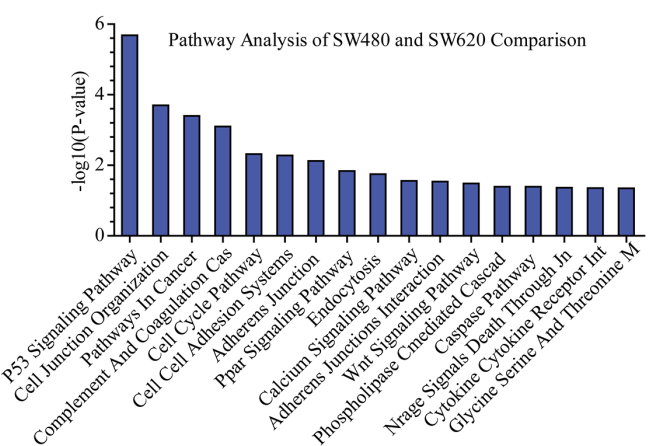

Pathway Analysis of Primary CRC and Liver Metastases Comparison

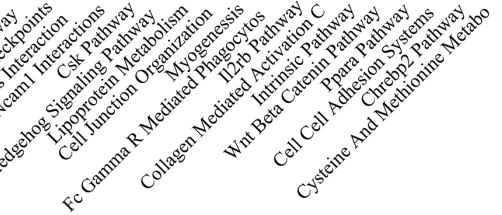

Figure 7: Comparison results of the differentially expressed genes between CRC primary-metastasis tissues and SW480SW620 cell lines. A. Overlapping and nonoverlapping genes and keywords between CRC primary-metastasis tissues and SW480-SW620 cell lines. B. Pathway analysis of SW480 and SW620 comparison. C. Pathway analysis of primary CRC and liver metastases comparison. 
molecular leading to drug resistant ability enhancing in metastatic CRC cells.

\section{MATERIALS AND METHODS}

\section{Datasets}

The raw experimental data under accession no. GSE22834 used in the present study, contributed from Albert Y et al [45], is publically available in the Gene Expression Omnibus (GEO) database (http://www. ncbi. nlm.nih.gov/geo). These data, which include 31 primary CRC specimens from 30 patients (14 males and 16 females; age range: 36 to 80 ; stage $\mathrm{I} / \mathrm{II} / \mathrm{III} / \mathrm{IV}=1 / 10 / 11 / 8$, diagnosed between 2000 and 2004), and 32 CLM specimens from 31 patients (16 males and 15 females; age range: 40 to 82 ), were produced by cDNA microarrays. In the present study, using primary CRC specimens as control, the molecular variations in CLM specimens were identified by the GenCLiP 2.0 online tool [46] (http://ci.smu.edu.cn/).

\section{Patients and tissue samples}

Paraffin-embedded surgical specimens of human primary CRC specimens and corresponding lymph-node metastatic specimens (from Department of Pathology, Qingyuan People's Hospital, Jinan University) were used for clinical tissue microarray expression profiling analysis. We used 60 primary CRC specimens and corresponding lymph-node metastatic specimens from 60 patients (36 males and 24 females; age range: 30 to 85 ; Duke stage $\mathrm{III} / \mathrm{IV}=43 / 17$, diagnosed between 2005 and 2008). For the use of these clinical materials for research purposes, prior written informed consent and ethics approval were obtained from all participants and the Ethics Committees of the Qingyuan People's Hospital, respectively.

\section{Cell culture and chemicals}

Human colon adenocarcinoma SW480 and SW620 cells were obtained from American Type Culture Collection. The cells were cultured in RPMI 1640 medium (Gibco, USA) with 10\% fetal bovine serum (TBD, China) at $37^{\circ} \mathrm{C}$ under an atmosphere of $5 \% \mathrm{CO}_{2} .5-\mathrm{Fu}$ was purchased from Shanghai Xudong Haipu Pharmaceutical Co., Ltd., China.

\section{Apoptosis analysis}

For the apoptosis analysis, the cells were treated for $24 \mathrm{~h}$ with control or $5-\mathrm{Fu}(50 \mu \mathrm{mol} / \mathrm{L})$. The cells were measured using FACSAriaflow cytometer (BD Biosciences, USA), and Annexin V (+) cells were counted for apoptotic cells after Annexin V-fluorescein isothiocyanate/propidium iodide (FITC/PI)(BD Pharmingen, USA) double staining. The experiment was performed in triplicate.

\section{Immunofluorescence and confocal microscopy}

Cells were seeded onto glass cover-slips in 6-well plates at a density of $2 \times 10^{5} /$ well and incubated for $48 \mathrm{~h}$. The cells were fixed in acetone at $20^{\circ} \mathrm{C}$ for 30 $\min$. Then the cells were exposed to goat serum for 1 $\mathrm{h}$ and incubated with mouse anti- $\beta$-catenin $(1: 100)$ for $12 \mathrm{~h}$ at $4{ }^{\circ} \mathrm{C}$ and FITC-labelled rabbit-anti-mouse IgG (1:300, Dingguo Biotechnology, China) for $1 \mathrm{~h}$ at $37^{\circ} \mathrm{C}$. The cells were counterstained with 4', 6-diamidino-2phenylindole (DAPI) (Dingguo Biotechnology, China) for $10 \mathrm{~min}$ and analyzed using a laser scanning confocal microscope.

\section{Flow cytometry analysis of CD133 positive cell population}

Human colon adenocarcinoma SW480 and SW620 cell lines $\left(1 \times 10^{6}\right)$ were detached by treatment with $0.25 \%$ trypsin/EDTA and washed twice with phosphatebuffered saline. The cells were then resuspended in $100 \mu \mathrm{l}$ of Staining Buffer containing 1\% fetal bovine serum and place on ice for 20 min to block Fc receptors. After incubating with primary phycoerythrin antihuman CD133 antibody (Milteny, Germany) for another $10 \mathrm{~min}$ on ice in the dark, the cells were washed twice with 1 $\mathrm{ml}$ of ice-cold Staining Buffer and centrifuged $(300 \times \mathrm{g})$ for $10 \mathrm{~min}$ at $4{ }^{\circ} \mathrm{C}$. Cells resuspended in $0.3 \mathrm{ml}$ of $2 \%$ formaldehyde fixation buffer were analyzed using a FACSAriaflow cytometer and Cell Quest software (BD Biosciences). All flow cytometry results were obtained from two independent experiments performed in triplicate.

\section{Western blot analysis}

Following treatment with different drugs for $24 \mathrm{~h}$, the cells were collected and lysed. Protein content was measured by the BCA protein assay kit (Beyotime Institute of Biotechnology, Shanghai, China) and $20 \mu \mathrm{g}$ protein per lane was separated by $8 \%-12 \%$ sodium dodecyl sulfate polyacrylamide gel electrophoresis (SDS-PAGE) and transferred onto nitrocellulose membranes. Specific protein bands were achieved with an ECL detection reagent (Pierce, Rockford, IL, USA). Anti-CD44v6 (Cell Signaling Technology, Danvers, MA, USA) dilution was 1:1000. Anti- $\beta$-catenin (Cell Signaling Technology) dilutions were 1:1,000. Anti-MMP-9 and anti-TERT (Abcam, Cambridge, MA, USA) dilutions were1:500 and 1:800. Anti-EpCAM and anti- $\alpha$-tubulin (Cell Signaling Technology) dilutions were 1:500 and 1:1,000. Horseradish peroxidase (HRP)-conjugated goat-anti-rabbit and goat-anti-mouse IgG antibodies (ProteinTech Group, Chicago, IL, USA) dilutions were 1:3,000. $\alpha$-tubulin was used as a protein loading control. The images were captured with ChemiDocTM CRS+ Molecular Imager 
(Bio-Rad, Hercules, CA, USA). The density of the protein band was quantitated using Quantity One software (BioRad). The experiment was performed in triplicate.

\section{Rh123 efflux assay for P-gp function}

After exposed to 5 -Fu for $24 \mathrm{~h}$, the CRC cells were incubated with $4 \mu \mathrm{g} / \mathrm{ml}$ rhodamine 123 (Rh123) (KeyGEN Biotech. Co., Ltd, China) for 30 or $60 \mathrm{~min}$ at $37^{\circ} \mathrm{C}$. The fluorescence intensity of Rh123 in cells was detected by flow cytometry.

\section{Characterization of apoptosis morphology in cells}

Cells were plated in 96-well plates at 3,000 cells per well. After compound treatment, cells were fixed using $10 \%$ buffered formalin $/ 4 \%$ formaldehyde. Cellular DNA fragmentation morphology was detected by terminal deoxynucleotidyl transferase (TdT)-mediated nick end labeling (TUNEL) staining using ApopTag red in situ kit (Roche, AG., Germany) according to the manufacturer's directions. TUNEL-positive cells were visualized and analyzed using Cellomic ArrayScan II image analysis system (Cellomics, Inc., Pittsburgh, PA).

\section{Transwell migration and boyden chamber invasion assays}

For the transwell migration assay, $10^{5}$ cells in 100 $\mathrm{ml}$ of serum-free DMEM media were triplicate seeded in each fibronectin-coated polycarbonate membrane insert in a transwell apparatus (Corning). $600 \mu 1$ of $10 \%$ NCS in DMEM was added to the bottom chamber. SW480 and SW620 cells were incubated at $37^{\circ} \mathrm{C}$ for $12 \mathrm{~h}$. The inserts were washed twice with prewarmed PBS. Cells adhered on the lower surface were fixed with $100 \%$ methanol at RT for $15 \mathrm{~min}$ and stained with hematoxylin for $15 \mathrm{~min}$. Cell numbers in six predetermined fields in each replicate were counted under the microscope (Nikon ECLPSE 80i system; $\times 200$ ). All assays were independently repeated at least for three times. Cell invasion assays were performed as the migration assay except the transwell membrane was precoated with $24 \mathrm{mgml}^{-1}$ Matrigel (R\&D Systems) and the cells were incubated for 24 and $18 \mathrm{~h}$, respectively.

\section{TMA construction and immunohistochemistry}

For immunohistochemical staining (IHC), AntiCD133 (ZSBIO, Beijing, China) dilution was 1:100. Anti-CD44v6 (ZSBIO) dilution was 1:200. Anti-Cyclin D1 (ZSBIO) dilutions were 1:50. Anti-MRP (ZSBIO) dilutions were 1:50. Anti-P-gp (ZSBIO) dilutions were 1:50. Anti-TERT (Abcam, Cambridge, MA, USA) dilutions was 1:200. Anti- $\beta$-catenin (Fuzhou Maixin Biotech. Co., Ltd., Fujian, China) dilutions were 1:1. Anti-E-cadherin (ZSBIO) dilutions was 1:70. Anti-
EpCAM (Cell Signaling Technology, Danvers, MA, USA) dilutions was 1:800, and incubated overnight at $4{ }^{\circ} \mathrm{C}$. Chromogenic detection was then done using a peroxidase-conjugated secondary antibody and DAB reagents (ZSBIO). The proliferation index was analyzed using the Cyclin D1 antibody. Staining was measured as the percentage of positively stained nuclei in 200 tumor cells in a consecutive field. Counting was conducted on the most intensely stained areas. The immunostains were scored by two pathologists (FL and SGW) blinded to the clinical data.

\section{Statistical analysis}

Statistical analysis was performed with the SPSS13.0 software package (SPSS Inc., Chicago, IL, USA). Two-class Significance Analysis of Microarrays (SAM) was used to identify genes that were differentially expressed in 31 CRC specimens and 32 CLM specimens, as well as metastatic cells SW620 and primary CRC cells SW480, the statistical significance was assessed by a false discovery rate (FDR). Data are presented as mean $\pm \mathrm{SD}$. One way analysis of variance (ANOVA) was used for apoptosis, cell cycle and western blot data analyses. Comparison of the CSCs and drug resistance relative proteins between primary $\mathrm{CRC}$ and colorectal lymphatic metastasis specimens was performed with the related-samples Wilcoxon signed rank test and Spearman's correlation test. $p<0.05$ was considered to indicate a statistically significant difference.

\section{CONFLICTS OF INTEREST}

No potential conflicts of interest were disclosed.

\section{GRANT SUPPORT}

This study was sponsored by Guangdong Natural Science Foundation (no. 2014A030307007) and Sci-Tech Project Foundation of Qingyuan City (no. 2013A009).

\section{REFERENCES}

1. Torre LA, Bray F, Siegel RL, Ferlay J, Lortet-Tieulent J and Jemal A. Global cancer statistics, 2012. CA Cancer J Clin. 2015; 65:87-108.

2. Kouzminova N, Lu T and Lin AY. Molecular basis of colorectal cancer. N Engl J Med. 2010; 362:1245-1246, 1246-1247.

3. Reissfelder C, Rahbari NN, Koch M, Ulrich A, Pfeilschifter I, Waltert A, Muller SA, Schemmer P, Buchler MW and Weitz J. Validation of prognostic scoring systems for patients undergoing resection of colorectal cancer liver metastases. Ann Surg Oncol. 2009; 16:3279-3288. 
4. Ombrato L and Malanchi I. The EMT universe: space between cancer cell dissemination and metastasis initiation. Crit Rev Oncog. 2014; 19:349-361.

5. Dhillon AS and Tulchinsky E. FRA-1 as a driver of tumour heterogeneity: a nexus between oncogenes and embryonic signalling pathways in cancer. Oncogene. 2015; 34:4421-4428.

6. Arumugam T, Ramachandran V, Fournier KF, Wang H, Marquis L, Abbruzzese JL, Gallick GE, Logsdon CD, McConkey DJ and Choi W. Epithelial to mesenchymal transition contributes to drug resistance in pancreatic cancer. Cancer Res. 2009; 69:5820-5828.

7. Singh A and Settleman J. EMT, cancer stem cells and drug resistance: an emerging axis of evil in the war on cancer. Oncogene. 2010; 29:4741-4751.

8. Kerr D. Clinical development of gene therapy for colorectal cancer. Nat Rev Cancer. 2003; 3:615-622.

9. Quan D, Gallinger S, Nhan C, Auer RA, Biagi JJ, Fletcher GG, Law CH, Moulton CA, Ruo L, Wei AC and McLeod RS. The role of liver resection for colorectal cancer metastases in an era of multimodality treatment: a systematic review. Surgery. 2012; 151:860-870.

10. Shimada H, Tanaka K, Endou I and Ichikawa Y. Treatment for colorectal liver metastases: a review. Langenbecks Arch Surg. 2009; 394:973-983.

11. Lou Y, Preobrazhenska O, Auf DKU, Sutcliffe M, Barclay L, McDonald PC, Roskelley C, Overall CM and Dedhar S. Epithelial-mesenchymal transition (EMT) is not sufficient for spontaneous murine breast cancer metastasis. Dev Dyn. 2008; 237:2755-2768.

12. Yilmaz M and Christofori G. EMT, the cytoskeleton, and cancer cell invasion. Cancer Metastasis Rev. 2009; 28:15-33.

13. Baldus SE, Schaefer KL, Engers R, Hartleb D, Stoecklein $\mathrm{NH}$ and Gabbert HE. Prevalence and heterogeneity of KRAS, BRAF, and PIK3CA mutations in primary colorectal adenocarcinomas and their corresponding metastases. Clin Cancer Res. 2010; 16:790-799.

14. Brannon AR, Vakiani E, Sylvester BE, Scott SN, McDermott G, Shah RH, Kania K, Viale A, Oschwald DM, Vacic V, Emde AK, Cercek A, Yaeger R, Kemeny NE, Saltz LB and Shia J, et al. Comparative sequencing analysis reveals high genomic concordance between matched primary and metastatic colorectal cancer lesions. Genome Biol. 2014; 15:454.

15. McMullin RP, Wittner BS, Yang C, Denton-Schneider BR, Hicks D, Singavarapu R, Moulis S, Lee J, Akbari MR, Narod SA, Aldape KD, Steeg PS, Ramaswamy S and Sgroi DC. A BRCA1 deficient-like signature is enriched in breast cancer brain metastases and predicts DNA damage-induced poly (ADP-ribose) polymerase inhibitor sensitivity. Breast Cancer Res. 2014; 16:R25.

16. Yokobori T, Iinuma H, Shimamura T, Imoto S, Sugimachi K, Ishii H, Iwatsuki M, Ota D, Ohkuma M, Iwaya T, Nishida N, Kogo R, Sudo T, Tanaka F, Shibata K and Toh H, et al. Plastin3 is a novel marker for circulating tumor cells undergoing the epithelial-mesenchymal transition and is associated with colorectal cancer prognosis. Cancer Res. 2013; 73:2059-2069.

17. Nakagawa H, Liyanarachchi S, Davuluri RV, Auer H, Martin EJ, de la Chapelle A and Frankel WL. Role of cancer-associated stromal fibroblasts in metastatic colon cancer to the liver and their expression profiles. Oncogene. 2004; 23:7366-7377.

18. Palmieri V, Lucchetti D, Maiorana A, Papi M, Maulucci G, Calapa F, Ciasca G, Giordano R, Sgambato A and De Spirito M. Mechanical and structural comparison between primary tumor and lymph node metastasis cells in colorectal cancer. Soft Matter. 2015; 11:5719-5726.

19. Kubens BS and Zanker KS. Differences in the migration capacity of primary human colon carcinoma cells (SW480) and their lymph node metastatic derivatives (SW620). Cancer Lett. 1998; 131:55-64.

20. Chang PY, Chen JS, Chang NC, Chang SC, Wang MC, Tsai SH, Wen YH, Tsai WS, Chan EC and Lu JJ. NRAS germline variant G138R and multiple rare somatic mutations on APC in colorectal cancer patients in Taiwan by next generation sequencing. Oncotarget. 2016; doi: 10.18632/oncotarget.8885.

21. Zhang QQ, Zhou DL, Lei Y, Zheng L, Chen SX, Gou HJ, Gu QL, He XD, Lan T, Qi CL, Li JC, Ding YQ, Qiao L and Wang LJ. Slit2/Robo1 signaling promotes intestinal tumorigenesis through Src-mediated activation of the Wnt/ beta-catenin pathway. Oncotarget. 2015; 6:3123-3135. doi: 10.18632/oncotarget.3060.

22. Xu D, Yuan L, Liu X, Li M, Zhang F, Gu X, Zhang D, Yang Y, Cui B, Tong J, Zhou J and Yu Z. EphB6 overexpression and Apc mutation together promote colorectal cancer. Oncotarget. 2016; doi: 10.18632/oncotarget.9080.

23. Khan NP, Pandith AA, Hussain MU, Yousuf A, Khan MS, Wani KA and Mudassar S. Novelty of Axin 2 and lack of Axin 1 gene mutation in colorectal cancer: a study in Kashmiri population. Mol Cell Biochem. 2011; 355:149-155.

24. Suraweera N, Robinson J, Volikos E, Guenther T, Talbot I, Tomlinson I and Silver A. Mutations within Wnt pathway genes in sporadic colorectal cancers and cell lines. Int $\mathrm{J}$ Cancer. 2006; 119:1837-1842.

25. Wang FW, Wen L, Zhu SW, Yao Q, Cai YM and Ma G. [Mechanism of Wnt signaling pathway regulation by a truncated mutant of Axin2 in colorectal cancer]. [Article in Chinese]. Ai Zheng. 2007; 26:1041-1046.

26. Vermeulen L, De Sousa EMF, van der Heijden M, Cameron K, de Jong JH, Borovski T, Tuynman JB, Todaro M, Merz C, Rodermond H, Sprick MR, Kemper K, Richel DJ, Stassi $\mathrm{G}$ and Medema JP. Wnt activity defines colon cancer stem cells and is regulated by the microenvironment. Nat Cell Biol. 2010; 12:468-476.

27. Evans J, Essex A, Xin H, Amitai N, Brinton L and Griner E. Registered report: Wnt activity defines colon cancer stem cells and is regulated by the microenvironment. Elife. 2015; 4. 
28. Noda T, Nagano H, Takemasa I, Yoshioka S, Murakami M, Wada H, Kobayashi S, Marubashi S, Takeda Y, Dono K, Umeshita K, Matsuura N, Matsubara K, Doki Y, Mori M and Monden M. Activation of Wnt/beta-catenin signalling pathway induces chemoresistance to interferon-alpha/5fluorouracil combination therapy for hepatocellular carcinoma. Br J Cancer. 2009; 100:1647-1658.

29. Zhang X, Lou Y, Wang H, Zheng X, Dong Q, Sun J and Han B. Wnt signaling regulates the stemness of lung cancer stem cells and its inhibitors exert anticancer effect on lung cancer SPC-A1 cells. Med Oncol. 2015; 32:95.

30. Zhang ZM, Wu JF, Luo QC, Liu QF, Wu QW, Ye GD, She HQ and Li BA. Pygo2 activates MDR1 expression and mediates chemoresistance in breast cancer via the Wnt/betacatenin pathway. Oncogene. 2016.

31. Martins-Neves SR, Paiva-Oliveira DI, Wijers-Koster PM, Abrunhosa AJ, Fontes-Ribeiro C, Bovee JV, Cleton-Jansen $\mathrm{AM}$ and Gomes CM. Chemotherapy induces stemness in osteosarcoma cells through activation of Wnt/beta-catenin signaling. Cancer Lett. 2016; 370:286-295.

32. Zhao Y, Peng S, Jia C, Xu F, Xu Y and Dai C. Armc8 regulates the invasive ability of hepatocellular carcinoma through E-cadherin/catenin complex. Tumour Biol. 2016.

33. Huber AH and Weis WI. The structure of the beta-catenin/Ecadherin complex and the molecular basis of diverse ligand recognition by beta-catenin. Cell. 2001; 105:391-402.

34. Schmalhofer O, Brabletz $\mathrm{S}$ and Brabletz T. E-cadherin, beta-catenin, and ZEB1 in malignant progression of cancer. Cancer Metastasis Rev. 2009; 28:151-166.

35. Kase S, Sugio K, Yamazaki K, Okamoto T, Yano T and Sugimachi K. Expression of E-cadherin and beta-catenin in human non-small cell lung cancer and the clinical significance. Clin Cancer Res. 2000; 6:4789-4796.

36. Christofori $\mathrm{G}$ and Semb H. The role of the cell-adhesion molecule E-cadherin as a tumour-suppressor gene. Trends Biochem Sci. 1999; 24:73-76.

37. Tian X, Liu Z, Niu B, Zhang J, Tan TK, Lee SR, Zhao Y, Harris DC and Zheng G. E-cadherin/beta-catenin complex and the epithelial barrier. J Biomed Biotechnol. 2011; 2011:567305.
38. Hsu YM, Chen YF, Chou CY, Tang MJ, Chen JH, Wilkins RJ, Ellory JC and Shen MR. KCl cotransporter-3 downregulates E-cadherin/beta-catenin complex to promote epithelial-mesenchymal transition. Cancer Res. 2007; 67:11064-11073.

39. Onder TT, Gupta PB, Mani SA, Yang J, Lander ES and Weinberg RA. Loss of E-cadherin promotes metastasis via multiple downstream transcriptional pathways. Cancer Res. 2008; 68:3645-3654.

40. Conacci-Sorrell M, Zhurinsky J and Ben-Ze'Ev A. The cadherin-catenin adhesion system in signaling and cancer. J Clin Invest. 2002; 109:987-991.

41. Lu Z, Ghosh S, Wang Z and Hunter T. Downregulation of caveolin-1 function by EGF leads to the loss of E-cadherin, increased transcriptional activity of beta-catenin, and enhanced tumor cell invasion. Cancer Cell. 2003; 4:499-515.

42. Liu KP, Luo F, Xie SM, Tang LJ, Chen MX, Wu XF, Zhong $\mathrm{XY}$ and Zhao T. Glycogen Synthase Kinase 3beta Inhibitor (2'Z,3'E)-6-Bromo-indirubin- 3'-Oxime Enhances Drug Resistance to 5-Fluorouracil Chemotherapy in Colon Cancer Cells. Chin J Cancer Res. 2012; 24:116-123.

43. Wu X, Luo F, Li J, Zhong X and Liu K. Tankyrase 1 inhibitior XAV939 increases chemosensitivity in colon cancer cell lines via inhibition of the Wnt signaling pathway. Int J Oncol. 2016; 48:1333-1340.

44. Li J and Zhou BP. Activation of beta-catenin and Akt pathways by Twist are critical for the maintenance of EMT associated cancer stem cell-like characters. Bmc Cancer. 2011; 11:49.

45. Lin AY, Chua MS, Choi YL, Yeh W, Kim YH, Azzi R, Adams GA, Sainani K, van de Rijn M, So SK and Pollack JR. Comparative profiling of primary colorectal carcinomas and liver metastases identifies LEF1 as a prognostic biomarker. PLoS One. 2011; 6:e16636.

46. Wang JH, Zhao LF, Lin P, Su XR, Chen SJ, Huang LQ, Wang HF, Zhang H, Hu ZF, Yao KT and Huang ZX. GenCLiP 2.0: a web server for functional clustering of genes and construction of molecular networks based on free terms. Bioinformatics. 2014; 30:2534-2536. 